\title{
Rapid detection of laboratory cross-contamination with Mycobacterium tuberculosis using multispacer sequence typing Zoheira Djelouadji ${ }^{1}$, Jean Orehek ${ }^{2}$ and Michel Drancourt*1
}

\author{
Address: ${ }^{1}$ Unité de Recherche sur les Maladies Infectieuses et Tropicales Emergentes, UMR CNRS 6236, IRD 3R198, Université de la Méditerranée \\ IFR 48, Faculté de Médecine, Marseille, France and 2Département des Maladies Respiratoires, Hôpital Sainte-Marguerite, Assistance Publique- \\ Hopitaux de Marseille, Marseille, France \\ Email: Zoheira Djelouadji - zorreda@yahoo.fr; Jean Orehek - jean.orehek@mail.ap-hm.fr; \\ Michel Drancourt* - michel.drancourt@medecine.univ-mrs.fr \\ * Corresponding author \\ Published: 3 March 2009 \\ BMC Microbiology 2009, 9:47 doi:10.1 I86/I47|-2180-9-47 \\ Received: 16 June 2008 \\ Accepted: 3 March 2009 \\ (c) 2009 Djelouadji et al; licensee BioMed Central Ltd. \\ This is an Open Access article distributed under the terms of the Creative Commons Attribution License (http://creativecommons.org/licenses/by/2.0), \\ which permits unrestricted use, distribution, and reproduction in any medium, provided the original work is properly cited.
}

\begin{abstract}
Background: The ability to culture Mycobacterium tuberculosis from clinical specimens serves as the gold standard for the diagnosis of tuberculosis. However, a number of false-positive diagnoses may be due to cross-contamination of such specimens. We herein investigate such episode of cross-contamination by using a technique known as multispacer sequence typing (MST). This technique was applied to six $M$. tuberculosis isolates prepared within the same laboratory over a two-week period of time.
\end{abstract}

Results: MST analysis indicated a unique and common sequence profile between a strain isolated from a patient with proven pulmonary tuberculosis and a strain isolated from a patient diagnosed with lung carcinoma. Using this approach, we were able to provide a clear demonstration of laboratory cross-contamination within just four working days. Further epidemiological investigations revealed that the two isolates were processed for culture on the same day.

Conclusion: The application of MST has been demonstrated to serve as a rapid and efficient method to investigate cases of possible cross-contamination with $M$. tuberculosis.

\section{Background}

The isolation of Mycobacterium tuberculosis complex organisms from clinical specimens collected from suspected patients serves as the gold standard for the proper diagnosis of tuberculosis in the laboratory [1]. However, falsepositive cultures have been reported that result from the cross-contamination of specimens via a contaminated bronchoscope $[2,3]$ or, more often, by laboratory crosscontamination [4]. The latter situation has been reported at a frequency ranging from $0.1 \%$ to $3 \%$ of $M$. tuberculosis [1,4-8]. Laboratory cross-contamination should be suspected when M. tuberculosis is cultured from a smear-neg- ative specimen processed in the same batch as a culture from a smear-positive specimen. The factors that increase the likelihood of cross-contamination include instances when only one of several specimens from the same patient is culture-positive and instances when the clinician is considering a diagnosis other than tuberculosis, which the clinician believes to be more likely based on clinical observations [8]. Such false-positives resulting from cross-contaminated specimens are disadvantageous since, besides resulting in a misdiagnosis, they result in unnecessary treatment and delay further diagnostic investigations in an effort to derive a definitive and correct 
diagnosis [9]. Finally, these false-positive cultures lead to an overestimation of the incidence and prevalence of tuberculosis in humans [10].

A definitive demonstration of cross-contamination can be derived from precise molecular analyses of $M$. tuberculosis isolates. M. tuberculosis isolates harbouring identical genotypes are regarded as clones and are thus epidemiologically linked [11]. The most widely used technique for determining the genotype of $M$. tuberculosis is a technique known as IS6110-restriction fragment length polymorphism (RFLP) analysis. RFLP analysis requires a large amount of biological material and, thus, poses a risk to laboratory workers due to the harmful nature of this pathogen. Moreover, the latter method requires a substantial amount of time due to the fastidious nature of $M$. tuberculosis [12]. More importantly from, a strictly technical perspective, IS6110-RFLP analysis does a poor job of indicating the presence of $M$. tuberculosis when these organisms contain only a few copies of the IS6110 sequence [13]. Recently, the variable number tandem repeat (VNTR) PCR-based technique and the mycobacterial interspersed repetitive unit (MIRU) [14] technique have proven to be reliable methods for the resolution of cross-contamination events $[15,16]$.

We herein report the application of a new PCR-sequencing-based genotyping method, known as multispacer sequence typing (MST)[17], for determining whether specimens have been cross-contaminated with $M$. tuberculosis in the laboratory.

\section{Case report}

A 60-year-old man was admitted for an examination to determine whether he had interstitial pneumonia. The patient had been previously hospitalised for two weeks at a different location with symptoms that included shortness of breath, a fever of $38.5^{\circ} \mathrm{C}$, and a $7 \mathrm{~kg}$ loss of weight within the past month. At the aforementioned hospital, a chest radiograph indicated the presence of bilateral interstitial pneumonia. Subsequent microbiological investigations, including Ziehl-Neelsen staining and a PCR-based assay to test for the presence of $M$. tuberculosis on expectoration, indicated that there were no signs of such an infection. The patient was then transferred to our department for further evaluation. Clinical examination of the patient verified both a body temperature of $38-38.5^{\circ} \mathrm{C}$ and dyspnoea with $90 \%$ oxygen saturation under $6 \mathrm{~L} / \mathrm{min}$ oxygen. The medical history of the patient was unremarkable, except for previous treatment for arterial hypertension. The total body tomodensitometry indicated the presence of nodules in both lungs, in the mediastinal lymph nodes, and in a right axilar lymph node. The pertinent laboratory assays were performed and indicated a value of 5.9 leucocytes/ml with $76 \%$ polymorphonuclear cells and 190 platelets $/ \mathrm{ml}$. The erythrocyte sedimentation rate was determined to be $28 \mathrm{~mm}$ for the first hour. The lactate dehydrogenase level was $612 \mathrm{IU} / \mathrm{ml}$ (normal levels are < $430 \mathrm{IU} / \mathrm{ml}$ ), the gamma GT level was $699 \mathrm{IU} / \mathrm{ml}$ (normal levels are $<55 \mathrm{IU} / \mathrm{ml}$ ), the bilirubin concentration was 13 $\mu \mathrm{mol} / \mathrm{l}$, the AST level was $96 \mathrm{IU} / \mathrm{l}$ (normal values are $<25$ $\mathrm{IU} / \mathrm{ml}$ ), and the ALT level was shown to be $127 \mathrm{IU} / \mathrm{l}$ (normal values are $<45 \mathrm{IU} / \mathrm{ml}$ ). It was suspected that the patient had already begun to develop pulmonary tuberculosis and thus was recommended to receive anti-tuberculosis therapy since it was reported that $M$. tuberculosis was isolated from an expectoration that was collected 14 days prior during the first hospital visit. Due to the observation that the patient's respiratory status had worsened, the patient was admitted into an intensive care unit for a period of four days. The results of direct microscopic examinations using Gram and Ziehl-Neelsen staining of a surgical lung biopsy were negative. This sample, cultured in BACTEC (Becton and Dickinson, Le Pont de La Claix, France) and in 5\% blood agar in slant tubes (Labo Moderne, Dinan, France), remained sterile after a two-month incubation period. Subsequent histological examination discovered large B-cell lymphoma and further assessments confirmed that the patient had stage IV lymphoma that involved the lung, liver, and bone marrow. The patient then received the appropriate anti-lymphoma therapy.

\section{Results and Discussion}

Our investigation revealed isolation of a total of six $M$. tuberculosis strains from a laboratory that performed analyses for six different patients (including the index patient) within a 2-week period before and after the isolation of $M$. tuberculosis from the index patient (Figure 1). All isolates were recovered from respiratory tract specimens and identified as M. tuberculosis by phenotypic methods and the ETR-D sequencing method [18]. Isolate Tub1 (patient A) was recovered from a specimen received and handled on April $27^{\text {th }}$, while isolate Tub2 (patient B) was recovered from a specimen received on May $3^{\text {rd }}$, but handled for setting in culture on May $4^{\text {th }}$. Isolate Tub3 (index patient C) was recovered from a specimen received and handled on May $4^{\text {th }}$, while isolates Tub4, Tub5, and Tub6 (patients D, $\mathrm{E}$, and $\mathrm{F}$, respectively) were recovered from specimens received and handled on May $8^{\text {th }}$. Ziehl-Neelsen staining was performed on all six specimens and the subsequent analyses revealed the presence of acid-fast bacilli for all samples with the exception of the specimen collected from index patient $\mathrm{C}$, which exhibited no acid-fast bacillus. Epidemiological investigation indicated that patients $\mathrm{A}, \mathrm{D}$, and $\mathrm{E}$ resided in the same ward, whereas no epidemiological link was found between the other three patients, including index patient $\mathrm{C}$. 


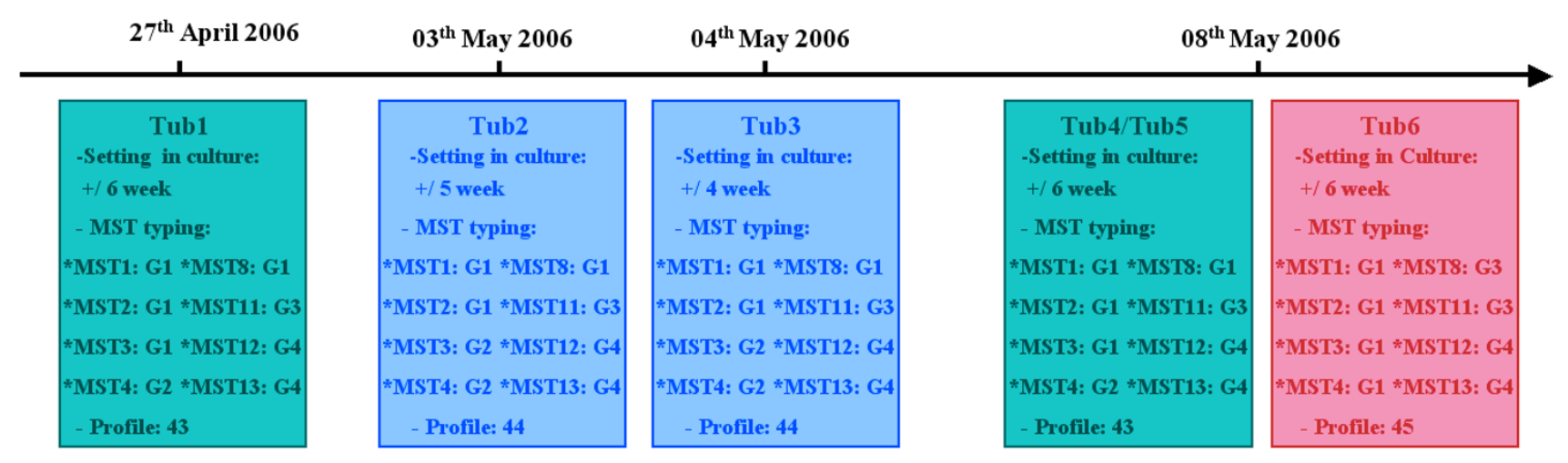

Figure I

Distribution of the MST profiles among M. tuberculosis isolates performed at different times in a laboratory.

Eight intergenic spacers were PCR amplified for each of the six M. tuberculosis isolates and yielded PCR products of the expected sizes. Sequences derived from these PCR products were combined and assembled for each of the six isolates. MST analysis was completed within four working days. Analysis of the sequence combinations determined three new genetic profiles, including profile ST43, which characterized the three isolates derived from patients $\mathrm{A}, \mathrm{D}$, and E; profile ST44, which characterized the two isolates derived from patient $\mathrm{B}$ and the index patient $\mathrm{C}$; and profile ST45, which was discovered in the isolate derived from patient $\mathrm{F}$ (Figure 1). These new profiles resulted from a novel combination of the following spacer alleles: the ST43 profile combined alleles 1/MST1, 1/MST2, 1/ MST3, 2/MST4, 1/MST8, 3/MST11, 4/MST12, and allele 4/ MST13; the ST44 profile combined alleles 1/MST1, 1/ MST2, 2/MST3, 2/MST4, 1/MST8, 3/MST11, 4/MST12, and allele 4/MST13; and the ST45 profile combined alleles 1/MST1, 1/MST2, 1/MST3, 1/MST4, 3/MST8, 3/ MST11, 4/MST12, and allele 4/MST13. The profiles for ST43, ST44, and ST45 have been added to our free and accessible MST database http://ifr48.timone.univ-mrs.fr/ MST Mtuberculosis/mst. MST genotyping data were assumed to be authentic based on the observations that the PCR-negative controls remained negative, coupled with the observation that all PCR products were of the predicted size. Moreover, analysis of the spacer sequences edited in this work identified three new profiles, clearly indicating that amplicons did not result from laboratory contamination as a consequence of previous experiments.

The MST genotyping data provided evidence to support epidemiological and clinical data that confirmed laboratory cross-contamination. Specifically, one profile (ST43) comprised three isolates recovered from epidemiologically-linked patients, whereas a different profile (ST45) characterized only one isolate from a specimen collected from an unrelated patient F. The profile ST44 was discovered for two $M$. tuberculosis isolates obtained from the index patient $\mathrm{C}$ and one unrelated patient $\mathrm{B}$. Microscopic examination of a respiratory tract specimen collected from patient $B$ indicated the presence of acid-fast bacilli, while the same analysis performed for a specimen from the respiratory tract of the index patient $\mathrm{C}$ showed no indication of acid-fast bacilli. Both of the latter two specimens were handled in the same laboratory, on the same day, and within the same batch of sample preparations, which explains the observation that the specimen recovered from the index patient (patient $C$ ) was contaminated by the specimen collected from patient B. Such a situation has been previously observed in cases of laboratory crosscontamination $[19,20]$. Interestingly, the frequency of false-positive cultures has been shown to be higher for laboratories that do not process high numbers of specimens [6], as was the case in the present report. As an example, in the laboratory setting, cross-contamination events may occur in the safety cabinet when a smear-positive specimen is handled in parallel with a smear-negative specimen, or during the phenotypic identification of isolates during the niacin test [15]. Cross-contamination of respiratory tract specimens by the avirulent $M$. tuberculosis H37Ra reference strain has also been reported [21].

The MST method, which was used in this study in addition to the more commonly used VNTR/MIRU typing method $[15,16]$, requires a relatively small amount of sample DNA from the patient. In contrast to the conventional IS6110-RFLP method, which requires a relatively large amount of DNA, both the MST and the VNTR/MIRU typing methods require only small DNA samples as they are based on PCR amplification of selected genomic regions [22]. The fact that such a small amount of material is handled during these aforementioned procedures is an obvious advantage, since it limits the risk of exposure of 
laboratory personnel to a dangerous pathogen. Since the MST method is based on sequence analysis, is reproducible and is easily exchangeable, we propose and offer a free and accessible M. tuberculosis MST database (at http:// ifr48.timone.univ-mrs.fr/MST_MTuberculosis/mst) so that microbiologists may compare the spacer sequence profiles they obtain with previously determined profiles for M. tuberculosis. The requirement for sequence analysis may limit the diffusion of MST to those laboratories that are equipped with an automatic sequencer, which is not a commonality in most laboratories, especially those in resource-limited countries.

Since MST uses PCR amplification as the first experimental step, it has the advantage of being applicable to DNA extracts from inactivated mycobacterial cultures [23] shortly after they are shown to be positive. The MST results were obtained in four working days (from the moment the culture was obtained to the interpretation of MST analysis). A similar, yet slightly longer delay of 13 days (median value) between initial analysis and interpretation of results was recently reported when using the VNTR/MIRU method. In contrast, the conventional IS6110 technique provided results in a median time of 45 days [16]. The delay period required to complete the MST analysis is certainly short enough to contribute to the interpretation of laboratory data that may have a significant clinical impact on patients.

\section{Conclusion}

Our report confirms the importance of rapid identification of cross-contamination. Indeed, the misdiagnosed patient received unnecessary anti-tuberculosis therapy and the final correct diagnosis was slightly delayed. MST typing proved to be an efficient new tool for the detection of cross-contamination with $M$. tuberculosis. In addition, MST results may be obtained within a few days, which significantly improves the quality of laboratory processing and, therefore, the quality of medical care for the patient.

\section{Methods \\ Epidemiological investigation}

We reviewed laboratory charts to identify mycobacterial isolates that were identified as M. tuberculosis during the 2week period before and after the isolation of M. tuberculosis in the index patient (total study period, 4 weeks). We carefully reviewed the batch number of each of these isolates in order to pinpoint the day on which clinical specimens were handled for setting in culture, as well as any epidemiological links between patients.

\section{Multispacer sequence typing}

Isolates were identified using conventional methods [24] and, after proper inactivation [23], by sequencing of the ETR-D spacer, as previously described [18]. The MST gen- otyping, PCR amplification and sequence analysis of eight intergenic spacers were performed as described previously [17]. Two negative controls consisting of the PCR mix in the absence of the target DNA template were also performed. Purified PCR products were sequenced by use of the BigDye Terminator 1.1 Cycle Sequencing kit (Applied Biosystems, Courtaboeuf, France). Sequencing electrophoresis was performed using a 3130 Genetic Analyser (Applied Biosystems). Sequences were aligned using CLUSTAL W http://pbil.ibcp.fr and compared to each other and with a local database of M. tuberculosis spacer sequences that is freely available on our website http:// ifr48.timone.univ-mrs.fr/MST Mtuberculosis/mst. This study was approved by the local Ethics Committee, Marseille, France.

\section{Competing interests}

The authors declare that they are the inventors of a protective patent on this matter deposited by the Mediterranée University, Marseilles, France.

\section{Authors' contributions}

DZ performed the described experiments, analysed the results and wrote the manuscript. JO performed the epidemiological investigation. MD analysed the results and contributed to drafting of the manuscript.

\section{Acknowledgements}

The authors would like to acknowledge the technical expertise of Christian Fontaine. This study was supported by Unité de Recherche sur les Maladies Infectieuses et Tropicales Emergentes, Marseille, France and Fuvre Antituberculeuse des Bouches du Rhône, Marseille, France.

\section{References}

I. Ruddy M, McHugh TD, Dale JW, Banerjee D, Maguire H, Wilson P, Drobniewski F, Butcher P, Gillespie SH: Estimation of the rate of unrecognized cross-contamination with Mycobacterium tuberculosis in London microbiology laboratories. J Clin Microbiol 2002, 40:4100-4104.

2. Larson JL, Lambert L, Stricof RL, Driscoll J, McGarry MA, Ridzon R: Potential nosocomial exposure to Mycobacterium tuberculosis from a bronchoscope. Infect Control Hosp Epidemiol 2003, 24:825-830.

3. Schoch OD, Pfyffer GE, Buhl D, Paky A: False-positive Mycobacterium tuberculosis culture revealed by restriction fragment length polymorphism analysis. Infection 2003, 31:189-191.

4. Small PM, McClenny NB, Singh SP, Schoolnik GK, Tompkins LS, Mickelsen PA: Molecular strain typing of Mycobacterium tuberculosis to confirm cross-contamination in the mycobacteriology laboratory and modification of procedures to minimize occurrence of false-positive cultures. J Clin Microbiol 1993, 3I:1677-1682.

5. Alland D, Kalkut GE, Moss AR, McAdam RA, Hahn JA, Bosworth W, Drucker E, Bloom BR: Transmission of tuberculosis in New York City. An analysis by DNA fingerprinting and conventional epidemiologic methods. N Engl J Med 1994, 330:1710-1716.

6. de Boer AS, Blommerde B, de Haas PE, Sebek MM, Lambregts-van Weezenbeek KS, Dessens M, van SD: False-positive Mycobacterium tuberculosis cultures in 44 laboratories in The Netherlands $(1993$ to 2000): incidence, risk factors, and consequences. J Clin Microbiol 2002, 40:4004-4009.

7. de CR, Soini H, Roscanni GC, Jaques M, Villares MC, Musser JM: Extensive cross-contamination of specimens with Mycobac- 
terium tuberculosis in a reference laboratory. J Clin Microbiol 1999, 37:916-919.

8. Martinez M, Garcia d V, Alonso M, Andres S, Bouza E, Cabezas T, Cabeza I, Reyes A, Sanchez-Yebra W, Rodriguez M, Sanchez MI, Rogado MC, Fernandez R, Penafiel T, Martinez J, Barroso P, Lucerna MA, Diez LF, Gutierrez C: Impact of laboratory cross-contamination on molecular epidemiology studies of tuberculosis. J Clin Microbiol 2006, 44:2967-2969.

9. Burman WJ, Stone BL, Reves RR, Wilson ML, Yang Z, el-Hajj H, Bates $\mathrm{JH}$, Cave MD: The incidence of false-positive cultures for Mycobacterium tuberculosis. Am J Respir Crit Care Med 1997, 155:321-326.

10. From the Centers for Disease Control and Prevention. Recall of isoniazid used for antimicrobial susceptibility testing for tuberculosis. JAMA 2000, 284:1642-1647.

II. Mathema B, Kurepina NE, Bifani PJ, Kreiswirth BN: Molecular epidemiology of tuberculosis: current insights. Clin Microbiol Rev 2006, I 9:658-685.

12. Miller AC, Sharnprapai S, Suruki R, Corkren E, Nardell EA, Driscoll JR, McGarry M, Taber H, Etkind S: Impact of genotyping of Mycobacterium tuberculosis on public health practice in Massachusetts. Emerg Infect Dis 2002, 8: I 285-I 289.

13. Barlow RE, Gascoyne-Binzi DM, Gillespie SH, Dickens A, Qamer S, Hawkey PM: Comparison of variable number tandem repeat and IS6 I IO-restriction fragment length polymorphism analyses for discrimination of high- and low-copy-number IS6 I I0 Mycobacterium tuberculosis isolates. J Clin Microbiol 200I, 39:2453-2457.

14. Loiez C, Willery E, Legrand JL, Vincent V, Gutierrez MC, Courcol RJ, Supply P: Against all odds: molecular confirmation of an implausible case of bone tuberculosis. Clin Infect Dis 2006 42:e86-e88.

15. Yan JJ, Jou R, Ko WC, Wu JJ, Yang ML, Chen HM: The use of variable-number tandem-repeat mycobacterial interspersed repetitive unit typing to identify laboratory cross-contamination with Mycobacterium tuberculosis. Diagn Microbiol Infect Dis 2005, 52:21-28.

16. Martin A, Herranz M, Lirola MM, Fernandez RF, Bouza E, Garcia d V: Optimized molecular resolution of cross-contamination alerts in clinical mycobacteriology laboratories. BMC Microbiol 2008, 8:30.

17. Djelouadji Z, Arnold C, Gharbia S, Raoult D, Drancourt M: Multispacer sequence typing for Mycobacterium tuberculosis genotyping. PLOS ONE 2008, 3:e2433.

18. Djelouadji Z, Raoult D, Daffe M, Drancourt M: A Single-Step Sequencing Method for the Identification of Mycobacterium tuberculosis Complex Species. PLoS Negl Trop Dis 2008, 2:e253.

19. Cook V], Stark G, Roscoe DL, Kwong A, Elwood RK: Investigation of suspected laboratory cross-contamination: interpretation of single smear-negative, positive cultures for Mycobacterium tuberculosis. Clin Microbiol Infect 2006, 12:1042-1045.

20. Carroll NM, Richardson M, van Helden PD: Criteria for identification of cross-contamination of cultures of Mycobacterium tuberculosis in routine microbiology laboratories. J Clin Microbiol 2003, 41:2269-2270.

21. Fitzpatrick L, Braden C, Cronin W, English J, Campbell E, Valway S, Onorato I: Investigation of Laboratory cross-contamination of Mycobacterium tuberculosis cultures. Clin Infect Dis 2004, 38:e52-e54.

22. Loiez C, Willery E, Legrand JL, Vincent V, Gutierrez MC, Courcol RJ, Supply P: Against all odds: molecular confirmation of an implausible case of bone tuberculosis. Clin Infect Dis 2006, 42:e86-e88.

23. Djelouagii Z, Drancourt M: Inactivation of cultured Mycobacterium tuberculosis organisms prior to DNA extraction. J Clin Microbiol 2006, 44: 1594-1595.

24. Pfyffer GE, Funke-Kissling P, Rundler E, Weber R: Performance characteristics of the BDProbeTec system for direct detection of Mycobacterium tuberculosis complex in respiratory specimens. J Clin Microbiol 1999, 37: I 37-I40.
Publish with Biomed Central and every scientist can read your work free of charge

"BioMed Central will be the most significant development for disseminating the results of biomedical research in our lifetime. "

Sir Paul Nurse, Cancer Research UK

Your research papers will be:

- available free of charge to the entire biomedical community

- peer reviewed and published immediately upon acceptance

- cited in PubMed and archived on PubMed Central

- yours - you keep the copyright
BioMedcentral 